\title{
Developing CRISPR/Cas9 technologies for research and medicine
}

\begin{abstract}
CRISPR/Cas, comprised of Clustered regularly interspaced short palindromic repeats (CRISPR) and associated proteins (Cas), provides adaptive immunity against foreign DNA in bacteria and archaea. Type II CRISPR/Cas9 has attracted considerable interest as a tool to probe and manipulate biological systems. It has been engineered to introduce genome editing in a simple, flexible, and efficient manner. Various approaches have been explored to reduce the off-target effects, which limit its applications in certain circumstances. Moreover, a catalytically inactive Cas9 was used to guide various effectors and a labeling agent to specific DNA targets through protein fusion, thus enabling genetic/epigenetic regulation and visualizing specific genomic loci in living cells. Further, CRISPR/Cas9 could have a significant impact on medicine by facilitating the generation of cell lines and animal models for therapeutic screening and evaluation, and by providing a new promising avenue for gene therapy.

CRISPR/Cas9 technologies are being rapidly developed in multiple directions, enabling new approaches for biological research. In addition, they may transform how drugs are developed and how human patients are treated in the future. Here we review CRISPR/Cas9 technologies originated, their applications utilizing the natural function, expansion of applications by repurposing to other functions, efforts for precision improvement, and finally how CRISPR/Cas9 may impact medicine.
\end{abstract}

Keywords: crispr/cas9, genome editing, genetic modulation, epigenetic modulation, genomic dna labeling, drug screening, animal models, gene therapy
Volume I Issue I - 2014

\author{
Yingze Zhao, Yue Ying, Yu Wang \\ State Key Laboratory of Reproductive Biology, Chinese \\ Academy of Sciences, China
}

\begin{abstract}
Correspondence: Yu Wang, State Key Laboratory of Reproductive Biology, Institute of Zoology, Chinese Academy of Sciences, Beijing 100101, China, Tel +86108261946I,
\end{abstract} Email wangyu@ioz.ac.cn

Received: May 29,2014 | Published: June 10,2014
Abbreviations: CRRNA, crispr rna; TRACRRNA, transactivating crrna; PAMs, protospacer adjacent motifs; HDR, homologydirected repair; DSB, double-strand breaks; ZF, zinc fingers; TALE, transcription activator-like effectors; ZFN, zinc-finger nuclease; NHEJ, non-homologous end-joining; dCas9, dead" cas9; CRISPRi, crispr inference; $\operatorname{sgRNA}$, single guide rna

\section{CRISPR/Cas9- inspiration from the mother nature}

The CRISPR/Cas system is immune defense machinery in bacteria and archaea that utilizes short RNAs to direct degradation of invading foreign nucleic acids, such as those from viruses or plasmids. ${ }^{1-3}$ This system essentially contains clustered regularly interspaced short palindromic repeats (CRISPRs) and their associated genes (Cas). ${ }^{2,4-7}$ CRISPR locus contains a series of short repeated sequences that are separated by non-repetitive spacer sequences derived from foreign genetic elements (Figure 1). This conserved repeat-spacer-repeat architecture was originally observed in the Escherichia coli genome in 1987. ${ }^{8}$ It was later found that the spacer sequences in CRISPR loci are identical to sequences in bacteriophage (phage) genomes and plasmids in 2005..$^{9-11}$ More than 40 different Cas proteins have been reported so far. ${ }^{12}$ Based on the sequences and structures of Cas protein, CRISPR/Cas system is primarily classified into three types I, II and III.6 In CRISPR/Cas system, invading foreign DNA is processed by Cas nuclease into small DNA fragments, which are then incorporated into CRISPR locus of host genomes as the spacers. In response to repeat viral / phage infections, the spacers are used as transcriptional templates for producing CRISPR RNA (crRNA), which guides Cas to target and cleave DNA sequences of invading viruses and phages ${ }^{13}$ (Figure 1). A unique feature of Class II is that a single Cas protein performs multiple functions whereas in other classes multiple Cas proteins are required to orchestrate and fulfill the role. ${ }^{13-16}$ Such simplicity makes it an ideal system as a biomedical research tool.

The type II CRISPR/Cas9 system is comprised of a long pre-crRNA transcribed from the spacer-repeat CRISPR locus, the multifunctional Cas9 protein, and a trans-activating crRNA (tracrRNA), which is important for processing the pre-crRNA and formation of the Cas 9 complex. TracrRNAs first hybridize to repeat regions of the pre-crRNA. Endogenous RNase III cleaves the hybridized crRNA-tracrRNAs, and an unknown mechanism removes the $5^{\prime}$ end of sequence in each spacer, yielding mature crRNAs that remain associated with both the tracrRNA and Cas9. Finally, the mature complex surrogates the foreign DNA and locates a target sequence and cuts both strands. ${ }^{17}$ The target sequence matches the protospacer and is adjacent to short sequences known as protospacer adjacent motifs (PAMs) at the $3^{\prime}$ end..$^{14,16}$ The PAM is an essential targeting component that serves as a self versus non-self recognition system to prevent the CRISPR locus itself from being targeted.18,19 The most commonly engineered system thus far, that of Streptococcus pyogenes, specifically recognizes the PAM sequence of NGG, where $\mathrm{N}$ can be any nucleotide. ${ }^{14,17,20}$ 


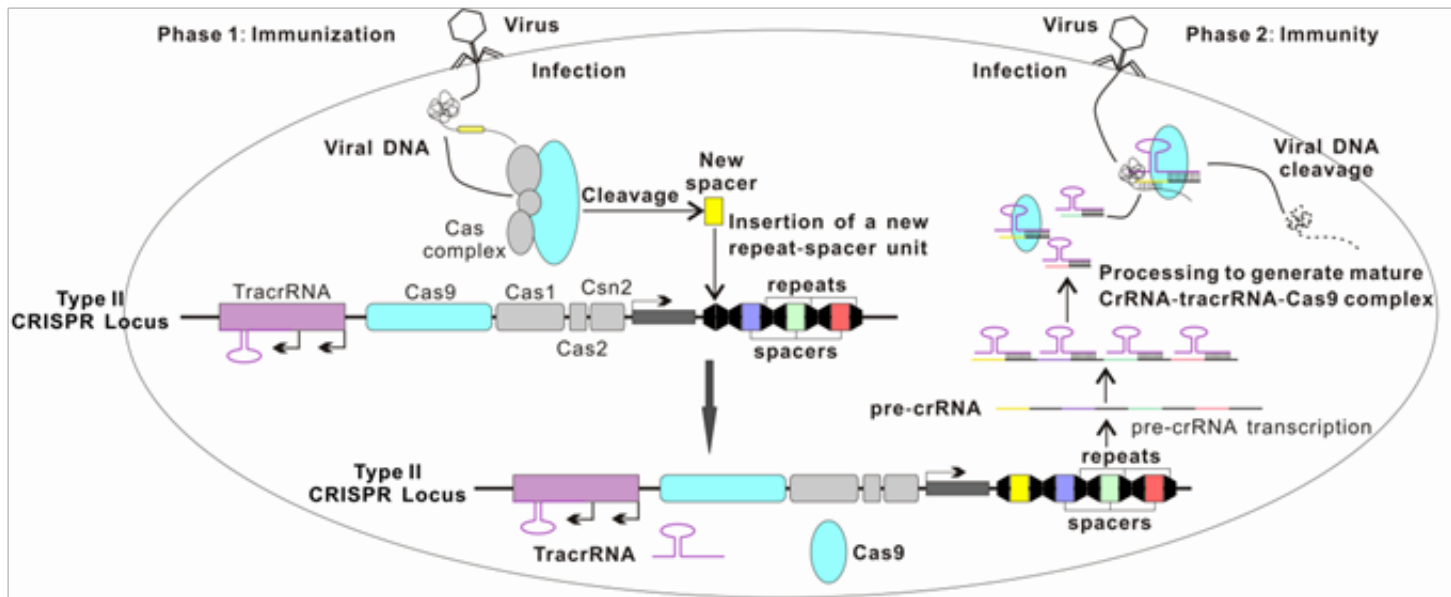

Figure I Mechanisms of CRISPR/Cas9 mediated bacterial immune-defense.

Phase I: Immunization process: the CRISPR locus contains a series of short repeated sequences that are separated by non-repetitive spacer sequences derived from foreign genetic elements. Invading foreign DNA from viruses and phages is processed by Cas nuclease into small DNA fragments, which are then incorporated into the CRISPR locus of host genomes as new spacers.

Phase 2: Immunity process: In response to viruses and phage infections, the CRISPR repeat-spacer array is transcribed into a pre-crRNA and processed into mature crRNAs, which guide Cas to cleave foreign DNA.

\section{CRISPR/cas9 mediated genome editing}

The ability of the CRISPR/Cas9 system to specifically target genomic sequence within living cells and organisms holds strong promise both as a powerful tool for biological research and as a potential avenue for gene therapy. ${ }^{21}$ For decades, traditional methods of gene targeting relied on homology-directed repair (HDR), a natural mechanism that cells use to repair double-strand breaks (DSB) in DNA. Mario Capecchi, Martin Evans and Oliver Smithies were awarded the Nobel Prize in 2007 for development of this technique. ${ }^{22}$ However, homologous recombination is often very inefficient without a DSB, thus targeting is always labor intensive with minimal success. To solve this problem, a DSB may be introduced at the targeting site, which triggers the recruitment of DNA repair machinery to the site, thus dramatically enhancing targeting efficiency. ${ }^{23,24}$
High specificity in the context of a genomic scale, ideally unique occurrence in the genome, needs to be achieved for precision in targeting. Most nucleases do not bind sequences that are long enough to reach high enough specificity, except a class named meganucleases. Therefore, meganucleases were engineered to facilitate genome editing via cutting DNA in a highly specific and targeted manner. ${ }^{25-27}$ Fusion constructs of non-specific nuclease and repetitive domains that confer DNA recognition specificity, including zinc fingers (ZF) and transcription activator-like effectors (TALE), have been developed and used in genome engineering. ${ }^{21}$ For example, the CCR5 gene was modified by a zinc-finger nuclease (ZFN) in CD4 T cells of human AIDS patients, which are then infused autologously to decrease the level of HIV infection. ${ }^{28}$ While all these are protein-based systems, which require significant effort in assembling the constructs, CRISPR/ Cas9 system is simpler, more flexible, effective and confers DNA recognition specificity by RNA (Table 1 ).

Table I Comparison of CRISPR/Cas9,TALE, ZF and Mega nuclease technologies

\begin{tabular}{|c|c|c|c|c|}
\hline & CRISPR/Cas9 & TALE & $\mathbf{Z F}$ & Mega nuclease \\
\hline $\begin{array}{l}\text { Type of } \\
\text { recognition }\end{array}$ & RNA-DNA & Protein-DNA & Protein-DNA & Protein-DNA \\
\hline $\begin{array}{l}\text { Off-target } \\
\text { effects }\end{array}$ & $\begin{array}{l}\text { More potential off- } \\
\text { target effects than } \\
\text { TALEs, ZFs and Mega } \\
\text { nuclease }\end{array}$ & $\begin{array}{l}\text { Less observed off-target effects } \\
\text { than CRISPR/Cas } 9\end{array}$ & $\begin{array}{l}\text { More potential off-target effects than } \\
\text { TALEs }\end{array}$ & Potential off-target effects \\
\hline Multiplexing & Capable and easy & Labor intensive and Rarely used & Labor intensive and Rarely used & Labor intensive and Rarely used \\
\hline $\begin{array}{l}\text { Target } \\
\text { sequence }\end{array}$ & $\begin{array}{l}\text { Adjacent to PAM, a } \\
\text { short sequence varies } \\
\text { among CRISPR/Cas9s } \\
\text { from different species }\end{array}$ & $\begin{array}{l}\text { Each TAL repeat binds a base pair } \\
\text { of DNA. Sequences targeted by } \\
\text { TAL effector repeats are typically } \\
\text { directly preceded by a thymine } \\
\text { (T) that is required for maximal } \\
\text { activity }\end{array}$ & $\begin{array}{l}\text { Each zinc finger binds a 3bp DNA target. } \\
\text { Not all } 3 \text { bp sequences can be targeted. } \\
\text { Assembly of } 3-4 \text { zinc finger modules is } \\
\text { required for specificity in recognition }\end{array}$ & $\begin{array}{l}\text { Only pre existing mega } \\
\text { nuclease recognition site. The } \\
\text { absence of that same site from } \\
\text { the targeting vector is required }\end{array}$ \\
\hline $\begin{array}{l}\text { Applications } \\
\text { in research }\end{array}$ & $\begin{array}{l}\text { I. Genome editing; } \\
\text { 2. Genetic modulation; } \\
\text { 3. Dynamic Imaging of } \\
\text { genomic loci; }\end{array}$ & $\begin{array}{l}\text { I. Genome editing; } \\
\text { 2. Genetic and epigenetic } \\
\text { modulation; }\end{array}$ & $\begin{array}{l}\text { I. Genome editing; } \\
\text { 2. Genetic modulation; }\end{array}$ & I. Genome editing; \\
\hline $\begin{array}{l}\text { Applications } \\
\text { in clinic }\end{array}$ & & & $\begin{array}{l}\text { Gene editing of CCR5 in autologous } \\
\text { CD4+T cells of persons infected with HIV }\end{array}$ & \\
\hline
\end{tabular}


The underlining principle for all the new genome editing technologies is that the introduced DSBs are repaired by either nonhomologous end-joining (NHEJ) ${ }^{29}$ without homologous DNA or more precise HDR in its presence ${ }^{30}$ (Figure 2A). NHEJ is an errorprone process that is often accompanied by insertion or deletion of nucleotides (indels) at the targeted site, sometimes resulting in a genetic knockout of the targeted region of the genome due to frameshift mutations or the insertion of a premature stop codon. ${ }^{31}$ Moreover, Cas9-induced DNA breaks can promote efficient rearrangement between pairs of targeted loci over a long distance through the nonhomologous end-joining (NHEJ) pathway of DSB repair. ${ }^{32}$ Cas9 has been used to modify genome sequence through NHEJ in bacteria, ${ }^{33}$ in cultured human cancer cell lines and human induced pluripotent stem cells, ${ }^{34-37}$ as well as in whole organisms, including zebrafish, mouse, and monkey. ${ }^{38-43}$ In the presence of homology DNA of the target site, HDR also occurs so that host genome DNA between homologous arms is exchanged out and donor DNA is inserted. Such approach has been used for genome engineering with high precision, from introducing single nucleotide mutations to introducing new genes to specific locations in the genome. ${ }^{31,44}$ As an example, two basepair substitutions of Tet genes can be introduced into the mouse genome via zygote microinjection of Cas9 mRNA, gRNA, and oligo donors. ${ }^{38}$

Following the initial demonstrations that type II CRISPR/Cas9 could be programmed to cut various DNA sites in bacteria, ${ }^{14}$ a series of reports subsequently showed that CRISPR/Cas9 systems can be engineered to introduce genome editing in a wide variety of species including crop, zebrafish, mouse, rat, rabbit, pig and monkey. ${ }^{38-43,45-50}$ Moreover, CRISPR/Cas9 based genome editing can be easily multiplexed by introducing multiple gRNAs..$^{35,38,50,51}$ Perhaps the best example for utilizing this great flexibility is the functional genomics screens with a large pool of gRNAs (Figure 2A). ${ }^{52-55}$

\section{Reducing the off-target effects of crispr/cas?}

Although CRISPR/Cas9 demonstrated obvious advantages for genomic modifications in biology, high-frequency off-target effects are the bottleneck for some applications. ${ }^{56-59}$ Off-target cleavages are one major concern in CRISPR/Cas9-mediated genome editing. The repair of off-target DSBs could result in deletions, inversions, translocations, and unknown off-target mutations, which may lead to undesired activation of oncogenes or inactivation of tumor suppressor genes. Several studies investigated the patterns of off-targeting effects and discovered that mismatches are generally better tolerated at the $5^{\prime}$ end of the 20-nt targeting region of the gRNA than at the $3^{\prime}$ end. ${ }^{14,32,34}$ The $8-12 \mathrm{bp}$ at the $3^{\prime}$ ' end of the targeting sequence, known as the 'seed' sequence, are crucial for target recognition in vitro and in bacterial cells..$^{14,33,35,60,61}$ However, the effects of mismatches are not always predictable based on their location within the gRNA targeting region; some mismatches in the $5^{\prime}$ end may have dramatic effects, whereas some in the $3^{\prime}$ end do not obviously affect CRISPR/ Cas9 activity. ${ }^{56}$ In addition, not all nucleotide substitutions at a given position necessarily confer equivalent effects on activity. ${ }^{62}$

Various approaches were explored to reduce the off-target mutagenic effects of CRISPR/Cas9. One strategy is to use paired "nickases". Cas9 variants that cut one strand rather than both strands of the target DNA sites known as "nickases". Because individual nicks in the genome are repaired with high fidelity, simultaneous nicking via appropriately offset guide RNAs is required for double-stranded breaks and extends the number of specifically recognized bases for target cleavage. The paired nickases targeted to sites on opposite DNA strands separated by 4 to $100 \mathrm{bp}$ can efficiently introduce both indel mutations and HDR events with a single-stranded DNA oligonucleotide donor template in mammalian cells. ${ }^{57,59,63,64}$ Another proposed approach for reducing Cas9-induced off-target effects of gRNAs in human cells involves the use of truncated gRNAs. ${ }^{65}$ These truncated gRNAs with a shortened $5^{\prime}$ end are 17 or 18 nucleotide long. They generally function as efficiently as full-length gRNAs in directing on-target Cas9 activity but show decreased undesired mutagenic effects at off-target sites by 5,000-fold or more. ${ }^{65}$ Recently, an approach was also used to improve DNA cleavage specificity by fusions of catalytically inactive Cas 9 and FokI nuclease (fCas9). ${ }^{66}$ Two fCas9 monomers simultaneously bind target sites 15 or 25 base pairs apart. FokI nuclease cleaves DNA only if the target sites are occupied simultaneously by two FokI domains at a specified distance and in a specific half-site orientation. It was reported that fCas9 modified target DNA sites with $>140$-fold higher specificity than wild-type Cas 9 and with an efficiency similar to that of paired Cas9 'nickases'. ${ }^{66}$

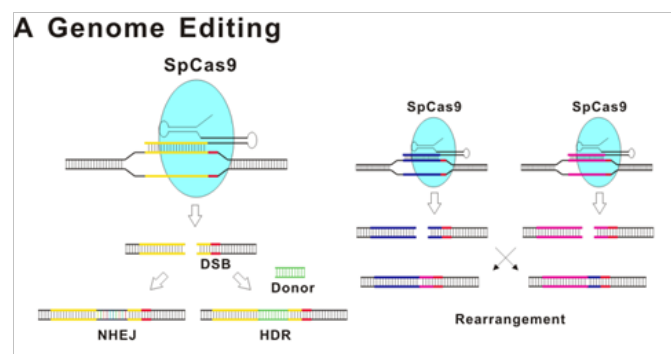

B Genetic/Epigenetic Modulation

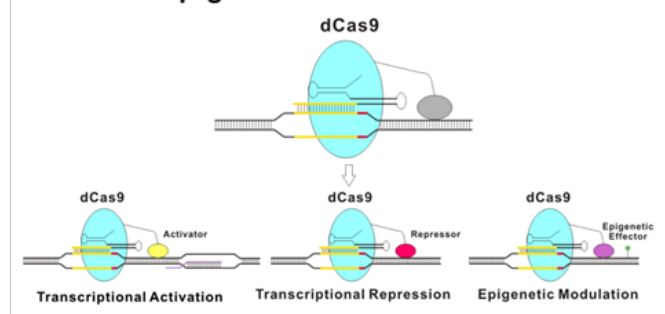

C Dynamic Imaging of Genomic Loci

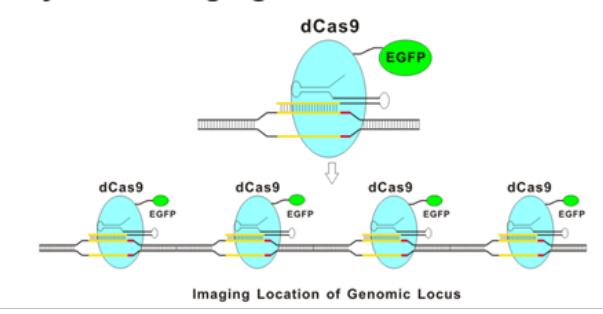

Figure 2 Overview of CRISPR/Cas9 applications.

(A) Genome editing: CRISPR/Cas9 is used to generate double strand breaks (DSB) at specific sites. The introduced DSBs are repaired by either non-homologous end-joining (NHEJ) or homology-directed repair (HDR) (the cartoon on the left). Efficient rearrangement over a long distance in the genome can also be promoted between pairs of targeted loci (the cartoon on the right).

(B) Genetic/epigenetic modulation: a transcriptional activation domain or a transcriptional repression domain fused with a catalytically inactive Cas9, dCas9, implements precise and stable transcriptional control of target genes. Epigenetic effectors fused with dCas9 could alter epigenetic states of the target loci, such as histone modifications or DNA methylation.

(C) dCas9 was fused with a fluorescent protein (Enhanced Green Fluorescent Protein, EGFP) to visualize specific genome loci in live cells. 


\section{Repurposing crispr/cas9}

\section{Genetic/epigenetic modulation}

The CRISPR/Cas9 system has the potential to regulate endogenous gene expression. Catalytically inactive or "dead" Cas9 (dCas9) bearing mutations at the two cleavage sites renders Cas9 unable to cut DNA, but it can still be recruited by gRNAs to target specific DNA sites within the genome. ${ }^{14,21,67}$ Therefore, dCas9 with heterologous effector domain functions can be recruited to specific genome loci, thus modulating the genetic/epigenetic states (Figure 2B). dCas9 fusions to a transcriptional activation domain (e.g. VP64 and the p65 subunit of nuclear factor kappa B; NF- $\mathrm{kB}$ ) or a transcriptional repression domain (e.g. the Krüppel-associated box (KRAB) domain) implements precise and stable transcriptional control of target genes. ${ }^{68-72}$ For example, single or multiple gRNAs can direct a VP64 transcriptional activation domain fused with a dCas9 protein to activate the expression of several endogenous genes. ${ }^{68,69}$ An alternative strategy of using aptamer fused with gRNA for binding an effector domain has also been reported. ${ }^{64}$ Although activity with this strategy is less robust than direct fusions to dCas9, this type of configuration might provide additional options and flexibility for recruitment of multiple effector domains. ${ }^{21}$ Interestingly, a synergistic effect of multiple gRNAs to target the same promoter for more effective modulation was observed in multiple studies. ${ }^{64,68,69,71}$

A modified CRISPR/Cas9 system named CRISPR inference (CRISPRi) has been developed for regulation of gene expression in eukaryotic cells. ${ }^{73}$ The CRISPRi system required that a catalytically inactive Cas9 protein was co-expressed with a customizable single guide RNA (sgRNA) to form a recognition complex, which interferes with transcriptional elongation and the binding of RNA polymerase and transcription factor. However, the degree of repression achieved by CRISPRi is modest in mammalian cells. ${ }^{73}$

Recently, several laboratories reported that epigenetic modifications of DNA and chromatin can be introduced via fusing epigenetic effectors with TAL repeats. ${ }^{70,74,75} \mathrm{~A}$ dCas 9 fusion might also be used to perform targeted 'epigenetic modulation'. Such targeted perturbation of the epigenetic status of specific loci would allow, for the first time, functional dissection of causative events among candidates found in association studies.

\section{Dynamic imaging of genomic loci}

d Cas9 fused with a fluorescent protein have been used to visualize specific genome loci in live cells (Figure 2C). ${ }^{76}$ The movements of telomeres labeled by dCas9::GFP or TRF1, a major telomerebinding protein, were characterized. No significant difference was observed between these two labeling methods, thus supporting dCas9 as a neutral probe of genomic DNA. This labeling strategy, among others, provides a useful tool for studying chromosome dynamics and structure and further extends dCas9-based applications.

\section{Potential clinical applications of crispr/cas9}

Given the tremendous value of CRISPR/Cas9 as a versatile tool to modulate various aspects of biological systems, it may provide equally exciting opportunities in clinical applications in addition to those in basic biological research.

There are two avenues ahead that CRISPR/Cas9 technologies could impact clinical translation. First, CRISPR/Cas9 could dramatically accelerate the pace for generating cell lines and animal models that harbor desired disease genes and reporters, ${ }^{41,42,48-50,77,78}$ which are used for drug screening and preclinical evaluation. CRISPR/Cas9 would be even more empowering when partnered with other transforming technologies such as iPS cell reprogramming and haploid stem cell targeting. ${ }^{49,79-87}$ Second, CRISPR/Cas9 itself might provide a new avenue for gene therapy. The success of ZFN in treating AIDS in a human clinical trial brought a lot of excitement and hope for treating a wide-variety of human diseases using similar approaches including CRISPR/Cas9. ${ }^{28}$ Considering the versatility of CRISPR/Cas9 systems, one can envision multiple ways for it to be applied in clinic one day, including genetic correction, ${ }^{42,77}$ epigenetic correction, and modulation of "disease" gene expression. ${ }^{78}$

To make the utility of CRISPR/Cas9 a reality in clinic, scientists first need to engineer the CRISPR/Cas9 system to a much higher level. First, precision of this technology needs to be further optimized and off-target effects need to be eliminated or controlled in order to ensure safety. Second, effective delivery of the CRISPR/Cas9 components, especially using non-integrative delivery technologies, shall be developed and matured. Third, the versatility of Cas9 to target any DNA sequence would need to be achieved which rely on the discovery of more orthogonal systems from nature or the development of Cas9 variants in laboratories that recognize distinct PAMs. ${ }^{88}$

\section{Acknowledgements}

Work in Y.W.'s laboratory is supported by the National Basic Research Program of China (Grant No. 2014CB964900) and funding from the Chinese Academy of Sciences.

\section{Conflict of interest}

The author declares no conflict of interest.

\section{References}

1 Bhaya D, Davison M, Barrangou R. CRISPR-Cas systems in bacteria and archaea: versatile small RNAs for adaptive defense and regulation. Annu Rev Genet. 2011;45:273-297.

2 Wiedenheft B, Sternberg SH, Doudna JA. RNA-guided genetic silencing systems in bacteria and archaea. Nature. 2012;482(7385):331-338.

3 Horvath P, Barrangou R. CRISPR/Cas, the immune system of bacteria and archaea. Science. 2010;327(5962):167-170.

4 Fineran PC, Charpentier E. Memory of viral infections by CRISPR-Cas adaptive immune systems: acquisition of new information. Virology. 2012;434(2):202-209.

5 Marraffini LA, Sontheimer EJ. CRISPR interference: RNAdirected adaptive immunity in bacteria and archaea. Nat Rev Genet. 2010;11(3):181-190.

6 Makarova KS, Haft DH, Barrangou R, et al. Evolution and classification of the CRISPR-Cas systems. Nat Rev Microbiol. 2011;9(6):467-477.

7 Sorek R, Lawrence CM, Wiedenheft B. CRISPR-mediated adaptive immune systems in bacteria and archaea. Annu Rev Biochem. 2013;82:237-266.

8 Ishino Y, Shinagawa H, Makino K, et al. Nucleotide sequence of the iap gene responsible for alkaline phosphatase isozyme conversion in Escherichia coli, and identification of the gene product. $J$ Bacteriol. 1987;169(12):5429-5433.

9 Bolotin A, Quinquis B, Sorokin A, et al. Clustered regularly interspaced short palindrome repeats (CRISPRs) have spacers of extrachromosomal origin. Microbiology. 2005;151(Pt 8):2551-2561.

10 Mojica FJ, Diez-Villasenor C, Garcia-Martinez J, et al. Intervening sequences of regularly spaced prokaryotic repeats derive from foreign genetic elements. J Mol Evol. 2005;60(2):174-182. 
11 Pourcel C, Salvignol G, Vergnaud G. CRISPR elements in Yersinia pestis acquire new repeats by preferential uptake of bacteriophage DNA and provide additional tools for evolutionary studies. Microbiology. 2005;151(Pt 3):653-663

12 Haft DH, Selengut J, Mongodin EF, et al. A guild of 45 CRISPRassociated (Cas) protein families and multiple CRISPR/Cas subtypes exist in prokaryotic genomes. PLoS Comput Biol. 2005;1(6):e60.

13 Zhang F, Wen Y, Guo X. CRISPR/Cas9 for genome editing: progress implications and challenges. Hum Mol Genet. 2014;23(R1):R40-R46.

14 Jinek M, Chylinski K, Fonfara I, et al. A programmable dual-RNAguided DNA endonuclease in adaptive bacterial immunity. Science. 2012;337(6096):816-821.

15 Garneau JE, Dupuis ME, Villion M, et al. The CRISPR/Cas bacterial immune system cleaves bacteriophage and plasmid DNA. Nature. 2010;468(7320):67-71.

16 Sapranauskas R, Gasiunas G, Fremaux C, et al. The Streptococcus thermophilus CRISPR/Cas system provides immunity in Escherichia coli. Nucleic Acids Res. 2011;39(21):9275-9282.

17 Mali P, Esvelt KM, Church GM. Cas9 as a versatile tool for engineering biology. Nat Methods. 2013;10(10):957-963.

18 Marraffini LA, Sontheimer EJ. Self versus non-self discrimination during CRISPR RNA-directed immunity. Nature. 2010;463(7280):568-571.

19 Sashital DG, Wiedenheft B, Doudna JA. Mechanism of foreign DNA selection in a bacterial adaptive immune system. Mol Cell. 2012;46(5):606-615.

20 Deltcheva E, Chylinski K, Sharma CM, et al. CRISPR RNA maturation by trans-encoded small RNA and host factor RNase III. Nature. 2011;471(7340):602-607.

21 Sander JD, Joung JK. CRISPR-Cas systems for editing regulating and targeting genomes. Nat Biotechnol. 2014;32(4):347-355.

22 http://www.nobelprize.org/nobel_prizes/medicine/laureates/2007/

23 Johnson RD, Jasin M. Double-strand-break-induced homologous recombination in mammalian cells. Biochem Soc Trans. 2001;29(Pt2):196-201.

24 Sonoda E, Hochegger H, Saberi A, et al. Differential usage of nonhomologous end-joining and homologous recombination in double strand break repair. DNA Repair (Amst). 2006;5(9-10):1021-1029.

25 Rosen LE, Morrison HA, Masri S, et al. Homing endonuclease I-Cre derivatives with novel DNA target specificities. Nucleic Acids Res 2006;34(17):4791-4800

26 Arnould S, Chames P, Perez C, et al. Engineering of large numbers of highly specific homing endonucleases that induce recombination on novel DNA targets. J Mol Biol. 2006;355(3):443-458.

27 Smith J, Grizot S, Arnould S, et al. A combinatorial approach to create artificial homing endonucleases cleaving chosen sequences. Nucleic Acids Res. 2006;34(22):e149.

28 Tebas P, Stein D, Tang WW, et al. Gene editing of CCR5 in autologous CD4 T cells of persons infected with HIV. $N$ Engl J Med. 2014;370(10):901-910.

29 Lieber MR, Gu J, Lu H, et al. Nonhomologous DNA end joining (NHEJ) and chromosomal translocations in humans. Subcell Biochem. 2010;50:279-296.

30 Heyer WD, Ehmsen KT, Liu J. Regulation of homologous recombination in eukaryotes. Annu Rev Genet. 2010;44:113-139.

31 Wilkinson R, Wiedenheft B. A CRISPR method for genome engineering F1000Prime Rep. 2014;6:3.
32 Choi PS, Meyerson M. Targeted genomic rearrangements using CRISPR/ Cas technology. Nat Commun. 2014;5:3728.

33 Jiang W, Bikard D, Cox D, et al. RNA-guided editing of bacterial genomes using CRISPR-Cas systems. Nat Biotechnol. 2013;31(3):233239.

34 Mali P, Yang L, Esvelt KM, et al. RNA-guided human genome engineering via Cas9. Science. 2013;339(6121):823-826.

35 Cong L, Ran FA, Cox D, et al. Multiplex genome engineering using CRISPR/Cas systems. Science. 2013;339(6121):819-823.

36 Jinek M, East A, Cheng A, et al. RNA-programmed genome editing in human cells. Elife. 2013;2:e0471.

37 Cho SW, Kim S, Kim JM, et al. Targeted genome engineering in human cells with the Cas9 RNA-guided endonuclease. Nat Biotechnol. 2013;31(3):230-232.

38 Wang H, Yang H, Shivalila CS, et al. One-step generation of mice carrying mutations in multiple genes by CRISPR/Cas-mediated genome engineering. Cell. 2013;153(4):910-918.

39 Shen $\mathrm{B}$, Zhang $\mathrm{J}$, Wu H, et al. Generation of gene-modified mice via Cas9/RNA-mediated gene targeting. Cell Res. 2013;23(5):720-723.

40 Li D, Qiu Z, Shao Y, et al. Heritable gene targeting in the mouse and rat using a CRISPR-Cas system. Nat Biotechnol. 2013;31(8):681-683.

41 Niu Y, Shen B, Cui Y, et al. Generation of gene--modified cynomolgus monkey via Cas9/RNA-mediated gene targeting in one-cell embryos. Cell. 2014;156(4):836-843

42 Yin H, Xue W, Chen S, Bogorad RL, Benedetti E, et al. Genome editing with Cas9 in adult mice corrects a disease mutation and phenotype. Nat Biotechnol. 2014;32(6):551-553.

43 Hwang WY, Fu Y, Reyon D, et al. Efficient genome editing in zebrafish using a CRISPR-Cas system. Nat Biotechnol. 2013;31(3):227-229.

44 Jonnalagadda VS, Matsuguchi T, Engelward BP. Interstrand crosslinkinduced homologous recombination carries an increased risk of deletions and insertions. DNA Repair (Amst). 2005;4(5):594-605.

45 Shan Q, Wang Y, Li J, et al. Targeted genome modification of crop plants using a CRISPR-Cas system. Nat Biotechnol. 2013;31(8):686-688.

46 Xie K, Yang Y. RNA-guided genome editing in plants using a CRISPRCas system. Mol Plant. 2013;6(6):1975-1983.

47 Yang $\mathrm{D}, \mathrm{Xu} \mathrm{J}$, Zhu T, et al. Effective gene targeting in rabbits using RNA-guided Cas9 nucleases. J Mol Cell Biol. 2014;6(1):97-99.

48 Hai T, Teng F, Guo R, et al. One-step generation of knockout pigs by zygote injection of CRISPR/Cas system. Cell Res. 2014;24(3):372-375.

$49 \mathrm{Li} \mathrm{W}, \mathrm{Li} \mathrm{X}, \mathrm{Li} \mathrm{T}$, et al. Genetic modification and screening in rat using haploid embryonic stem cells. Cell Stem Cell. 2014;14(3):404-414.

50 Yang $H$, Wang $H$, Shivalila CS, et al. One-step generation of mice carrying reporter and conditional alleles by CRISPR/Cas-mediated genome engineering. Cell. 2013;154(6):1370-1379.

$51 \mathrm{Li} \mathrm{W}$, Teng F, Li T, et al. Simultaneous generation and germline transmission of multiple gene mutations in rat using CRISPR-Cas systems. Nat Biotechnol. 2013;31(8):684-686.

52 Wang T, Wei JJ, Sabatini DM, et al. Genetic screens in human cells using the CRISPR-Cas9 system. Science. 2014;343(6166):80-84.

53 Koike-Yusa H, Li Y, Tan EP, et al. Genome-wide recessive genetic screening in mammalian cells with a lentiviral CRISPR-guide RNA library. Nat Biotechnol. 2014;32(3):267-273.

54 Shalem O, Sanjana NE, Hartenian E, et al. Genome-scale CRISPR-Cas9 knockout screening in human cells. Science. 2014;343(6166):84-87. 
55 Zhou Y, Zhu S, Cai C, et al. High-throughput screening of a CRISPR Cas9 library for functional genomics in human cells. Nature. 2014;509(7501):487-491.

$56 \mathrm{Fu}$ Y, Foden JA, Khayter C, et al. High-frequency off-target mutagenesis induced by CRISPR-Cas nucleases in human cells. Nat Biotechnol. 2013;31(9):822-826

57 Shen B, Zhang W, Zhang J, et al. Efficient genome modification by CRISPR-Cas9 nickase with minimal off-target effects. Nat Methods. 2014;11(4):399-402.

58 Hruscha A, Krawitz P, Rechenberg A, et al. Efficient CRISPR/Cas9 genome editing with low off-target effects in zebrafish. Development. 2013;140(24):4982-4987.

59 Cho SW, Kim S, Kim Y, et al. Analysis of off-target effects of CRISPR Cas-derived RNA-guided endonucleases and nickases. Genome Res. 2014;24(1):132-141

60 Semenova E, Jore MM, Datsenko KA, et al. Interference by clustered regularly interspaced short palindromic repeat (CRISPR) RNA is governed by a seed sequence. Proc Natl Acad Sci U S A. 2011;108(25):10098-10103.

61 Wiedenheft B, Van Duijn E, Bultema JB, et al. RNA-guided complex from a bacterial immune system enhances target recognition through seed sequence interactions. Proc Natl Acad Sci U S A. 2011;108(25):1009210097.

62 Hsu PD, Scott DA, Weinstein JA, et al. DNA targeting specificity of RNA-guided Cas9 nucleases. Nat Biotechnol. 2013;31(9):827-832.

63 Ran FA, Hsu PD, Lin CY, et al. Double nicking by RNA-guided CRISPR Cas9 for enhanced genome editing specificity. Cell. 2013;154(6):13801389.

64 Mali P, Aach J, Stranges PB, et al. CAS9 transcriptional activators for target specificity screening and paired nickases for cooperative genome engineering. Nat Biotechnol. 2013;31(9):833-839.

$65 \mathrm{Fu} \mathrm{Y,} \mathrm{Sander} \mathrm{JD,} \mathrm{Reyon} \mathrm{D,} \mathrm{et} \mathrm{al.} \mathrm{Improving} \mathrm{CRISPR-Cas} \mathrm{nuclease}$ specificity using truncated guide RNAs. Nat Biotechnol. 2014;32(3):279284

66 Guilinger JP, Thompson DB, Liu DR. Fusion of catalytically inactive Cas9 to FokI nuclease improves the specificity of genome modification. Nat Biotechnol. 2014;32(6):577-582.

67 Gasiunas G, Barrangou R, Horvath P, et al. Cas9-crRNAribonucleoprotein complex mediates specific DNA cleavage for adaptive immunity in bacteria. Proc Natl Acad Sci U S A. 2012;109(39):E2579-2586.

68 Maeder ML, Linder SJ, Cascio VM, et al. CRISPR RNA-guided activation of endogenous human genes. Nat Methods. 2013;10(10):977979.

69 Perez-Pinera P, Kocak DD, Vockley CM, t al. RNA-guided gene activation by CRISPR-Cas9-based transcription factors. Nat Methods 2013;10(10):973-976.

70 Konermann S, Brigham MD, Trevino AE, et al. Optical control of mammalian endogenous transcription and epigenetic states. Nature. 2013;500(7463):472-476.

71 Cheng AW, Wang H, Yang H, et al. Multiplexed activation of endogenous genes by CRISPR-on an RNA-guided transcriptional activator system. Cell Res. 2013;23(10):1163-1171.
72 Gilbert LA, Larson MH, Morsut L, et al. CRISPR-mediated modular RNA-guided regulation of transcription in eukaryotes. Cell. 2013;154(2):442-451.

73 Larson MH, Gilbert LA, Wang X, et al. CRISPR interference (CRISPRi) for sequence-specific control of gene expression. Nat Protoc. 2013;8(11):2180-2196

74 Mendenhall EM, Williamson KE, Reyon D, et al. Locus-specific editing of histone modifications at endogenous enhancers. Nat Biotechnol. 2013;31(12):1133-1136

75 Maeder ML, Angstman JF, Richardson ME, et al. Targeted DNA demethylation and activation of endogenous genes using programmable TALE-TET1 fusion proteins. Nat Biotechnol. 2013;31(12):1137-1142.

76 Chen B, Gilbert LA, Cimini BA, et al. Dynamic imaging of genomic loci in living human cells by an optimized CRISPR/Cas system. Cell. 2013;155(7):1479-1491

77 Wu Y, Liang D, Wang Y, et al. Correction of a genetic disease in mouse via use of CRISPR-Cas9. Cell Stem Cell. 2013;13(6):659-662.

78 Schwank G, Koo BK, Sasselli V, et al. Functional repair of CFTR by CRISPR/Cas9 in intestinal stem cell organoids of cystic fibrosis patients. Cell Stem Cell. 2013;13(6):653-658.

79 Xue H, Wu J, Li S, et al. Genetic Modification in Human Pluripoten Stem Cells by Homologous Recombination and CRISPR/Cas9 System. Methods Mol Biol. 2016;1307:173-190.

80 Yang L, Guell M, Byrne S, et al. Optimization of scarless human stem cell genome editing. Nucleic Acids Res. 2013;41(19):9049-9061.

81 Horii T, Tamura D, Morita S, et al. Generation of an ICF syndrome model by efficient genome editing of human induced pluripotent stem cells using the CRISPR system. Int J Mol Sci. 2014;14(10):19774-19781.

82 Horii T, Morita S, Kimura M, et al. Genome engineering of mammalian haploid embryonic stem cells using the Cas9/RNA system. Peer $J$. 2013;1:e230.

83 Takahashi K, Tanabe K, Ohnuki M, et al. Induction of pluripotent stem cells from adult human fibroblasts by defined factors. Cell. 2007;131(5):861-872.

$84 \mathrm{Yu}$ J, Vodyanik MA, Smuga-Otto K, et al. Induced pluripotent stem cell lines derived from human somatic cells. Science. 2007;318(5858):19171920.

85 Takahashi K, Okita K, Nakagawa M, et al. Induction of pluripotent stem cells from fibroblast cultures. Nat Protoc. 2007;2(12):3081-3089.

86 Li W, Shuai L, Wan H, et al. Androgenetic haploid embryonic stem cells produce live transgenic mice. Nature. 2012;490(7420):407-411.

87 Yang H, Shi L, Wang BA, et al. Generation of genetically modified mice by oocyte injection of androgenetic haploid embryonic stem cells. Cell. 2012;149(3):605-617.

88 Esvelt KM, Mali P, Braff JL, et al. Orthogonal Cas9 proteins for RNAguided gene regulation and editing. Nat Methods. 2013;10(11):11161121. 